Hydrology and Earth System Sciences, 8(6), 1045-1050 (2004) C EGU

\title{
Statistical representation of mountain shading
}

\author{
Richard Essery \\ Centre for Glaciology, Institute of Geography and Earth Sciences, University of Wales, Aberystwyth SY23 3DB, UK \\ Email for author: rie@aber.ac.uk
}

\begin{abstract}
Shadows cast by the mountains themselves have a strong influence on the surface energy balance of mountainous regions. If the influence of shadows is to be included on sub-grid scales in a surface energy balance model, a statistical representation has to be used. Slope components calculated from digital elevation models of areas in North Wales and the French Alps are found to have double-exponential distributions. From this result, expressions are developed for the fractions of the areas that will be either self-shaded or shaded by remote topography as functions of solar elevation and time of day. These expressions are in good agreement with results from a terrain shading model.
\end{abstract}

Keywords: solar radiation, topography, surface energy balance, statistical parameterisation

\section{Introduction}

Solar radiation is a major component of the energy balance of the land surface. In mountainous regions, the amount of direct-beam solar radiation falling on the surface varies strongly with slope and aspect, introducing strong spatial variations in the surface energy balance (Barry, 1992). This, in turn, controls processes such as evaporation and snow melt, and influences distributions of vegetation, land use and glaciation. Spatial variations in illumination also influence the radiation received by remote-sensing instruments viewing complex topography. Many models for calculating distributions of solar radiation over topographic grids are available (e.g. Garnier and Ohmura, 1968; Dubayah and Rich, 1995; Kumar et al., 1997; Mckenney et al., 1999; Corripio, 2003). However, if variations in illumination over the surface are to be taken into account on sub-grid scales for surface energy balance models or on sub-pixel scales for interpretation of remotely sensed data, statistical characterisations have to be used.

Dubayah et al. (1990) developed parameterisations for the average and standard deviation of clear-sky solar radiation over gentle topography for solar elevations high enough that shadows do not occur. Shadows are more significant for mountainous topography (Oliphant et al., 2003), although variability due to extensive shading at low solar elevations is limited by the long optical paths. Ranzi and Rosso (1995) presented a method for calculating the average solar radiation over a basin, accounting for shadows, that involves integration around the perimeter of the basin rather than over a grid covering the interior. A point on the surface will be shaded if the angle of the surface away from the sun exceeds the solar elevation. Points that do not fulfil this criterion will still be in shadow if the sun lies below the local horizon, i.e. if the angle from the point to its horizon in the direction of the sun exceeds the solar elevation.

In this paper, a terrain shading model is used with digital elevation models (DEMs) to investigate topographic shading for mountainous areas in North Wales and the French Alps. Using statistical distributions of slope components calculated from the DEMs, expressions are developed for the fractions of the regions that are self-shaded or horizonshaded as functions of the solar elevation and the standard deviations of the slope components. These expressions are compared with results from the terrain shading model.

\section{Terrain shading}

Variations in direct-beam illumination across a valley for solar elevation $\theta$ are illustrated in Fig. 1. If $S_{0}$ is the radiation crossing a plane normal to the sun's rays, the radiation on a 


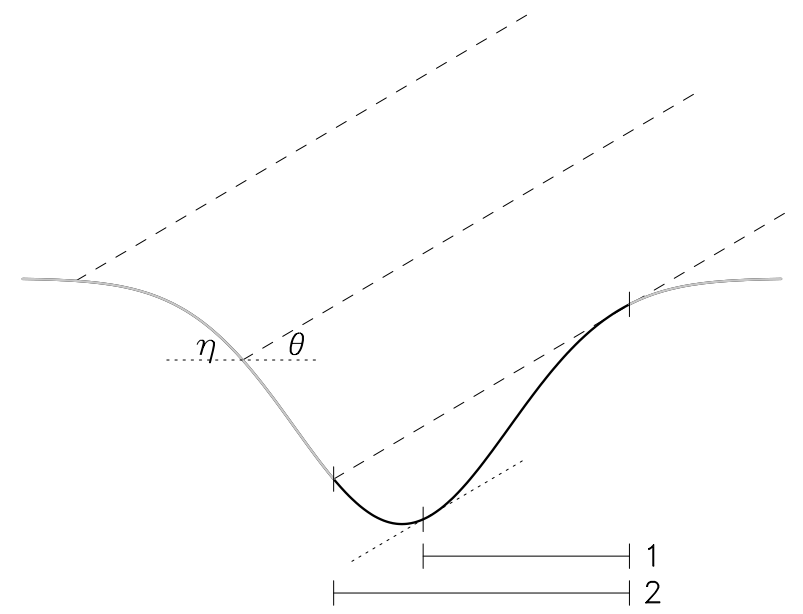

Fig. 1. Schematic illustration of variations in direct-beam solar illumination (dashed lines) across a valley; $\theta$ is the solar elevation and $\eta$ is the local slope angle. Region 1 is self-shaded and region 2 is shaded by the horizon.

sloping surface is $S_{0} \sin (\theta+\eta)$, where $\eta$ is the angle of the slope along the solar azimuth. If $\eta<-\theta$, as in region 1 of the surface in Fig. 1, this gives a negative quantity, and the direct-beam radiation is instead set to zero; such slopes are self-shaded. The sun lies below the local horizon for points in region 2, so this region of the surface lies in the shadow of remote topography.

For the hypothetical topography shown in Fig. 1, the selfshaded region is contained within the horizon-shaded region.
When slope and horizon angles are calculated on a finite grid, some cells may be classified as self-shaded but not horizon-shaded. In fact, this is an artefact due to the finite resolution of the grid. Only points just on the shaded side of ridge lines are self-shaded but not horizon-shaded. As the resolution is increased, the set of such points covers a vanishing fraction of the whole area, and the total shaded fraction predicted by a terrain shading model for clear skies converges to the predicted horizon-shaded fraction.

Terrain shading is calculated here using the model of Corripio (2003). Figures 2 (a) and (b) show $12 \mathrm{~km} \times 12 \mathrm{~km}$ areas in North Wales and the French Alps with the conventional but non-physical illumination from the northwest at a $45^{\circ}$ elevation. The DEMs from which these figures were derived both have a horizontal resolution of $50 \mathrm{~m}$. Shadow fractions calculated as fractions of the areas that are either horizon-shaded or both horizon-shaded and self-shaded differ by at most 0.04 for both DEMs.

North Wales is a classic area for glacial erosional landforms. The area shown in Fig. 2 (a) is centred on the summit of Snowdon $\left(1085 \mathrm{~m}, 53^{\circ} 5^{\prime} \mathrm{N}, 4^{\circ} 5^{\prime} \mathrm{W}\right)$, which is composed of Ordovician extrusive igneous rocks, with mudstones of similar age forming the lower slopes. The area was last uplifted and river valleys were carved out of the Snowdon massif in the Tertiary Period. Successive Quaternary ice sheets greatly modified the landscape but the last cirque glaciers disappeared after the Younger Dryas. Figure 2 (b), in contrast, shows a heavily glaciated area in the French Alps. The peak at the centre right of the image is the Aiguille Verte $\left(4122 \mathrm{~m}, 45^{\circ} 59^{\prime} \mathrm{N}, 6^{\circ} 26^{\prime} \mathrm{E}\right)$, with the Mer

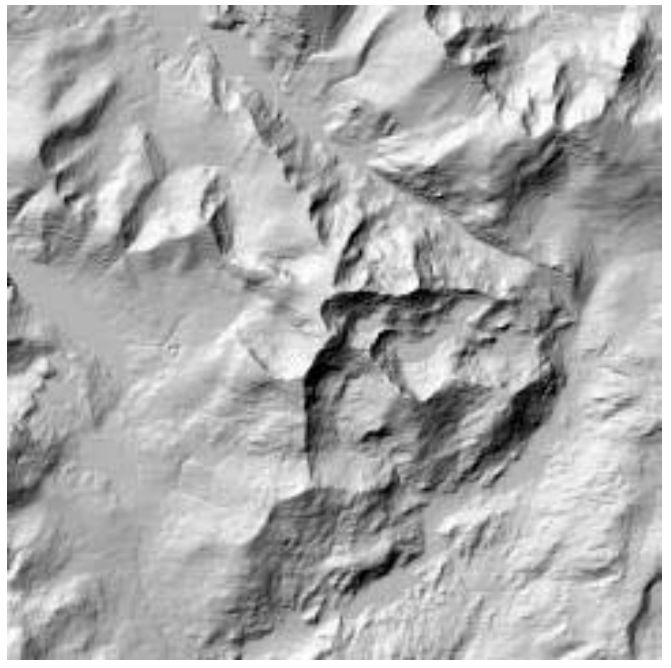

(a)

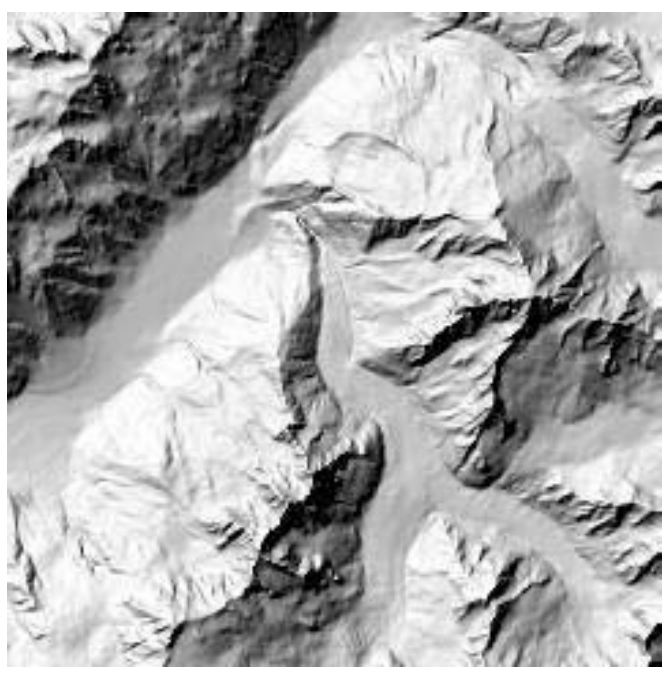

(b)

Fig. 2. Shaded topography for $12 \mathrm{~km} \times 12 \mathrm{~km}$ areas in (a) North Wales and (b) the French Alps, illuminated from the northwest with a solar elevation of $45^{\circ}$. 
de Glace glacier flowing northwards through the centre of the figure and the Chamonix valley lying towards the upper left.

\section{Statistical characterisation of shading and topography}

Direct-beam solar radiation on a surface depends on the slope and horizon angles for the surface, so a statistical characterisation will depend on their distributions. There are several methods for calculating slope components from DEMs (Skidmore, 1989). For example, the component of the slope (partial derivative of elevation) in the west-east direction can be approximated by the central difference

$$
\frac{\partial z}{\partial x} \approx \frac{z_{i+1, j}-z_{i-1, j}}{2 \Delta x},
$$

or by the average slope component of the two triangles defined by a grid cell boundary (Corripio, 2003),

$$
\frac{\partial z}{\partial x} \approx \frac{z_{i+1, j}+z_{i+1, j+1}-z_{i, j+1}-z_{i, j}}{2 \Delta x},
$$

where $z_{i, j}$ is the elevation of the cell in column $i$ and row $j$ of the DEM, and $\Delta x$ is the grid spacing. Equation (2) is used here for consistency with the terrain shading model but, although the choice of discretisation influences the patterns of calculated slope components (Corripio, 2003), it has little influence on their statistical distributions over large areas.

Slopes calculated by finite differences have average angles and standard deviations over the areas shown in Fig. 2 of $17^{\circ}$ and $11^{\circ}$ for the Snowdon DEM, and $29^{\circ}$ and $15^{\circ}$ for the Aiguille Verte DEM, but it should be noted that these values depend on the resolution of the DEMs (Dubayah et al., 1990). Frequency distributions for slope components in west-east and south-north directions are shown in Fig. 3. Plotted on a logarithmic probability scale in Fig. 3(a), distributions from the Snowdon DEM follow approximately straight lines for positive and negative slope components and so can be approximated by a double-exponential (Laplace) distribution,

$$
p(s)=\frac{1}{\sqrt{2} \sigma} \exp \left(-\frac{\sqrt{2}|s|}{\sigma}\right),
$$

where $s$ is the slope component and $\sigma$ is its standard deviation. Similar distributions were found for gentler topographies in the Canadian Arctic by Essery (2001) and Alaska by Bowling et al. (2004). There is more asymmetry in distributions, shown in Fig. 3 (b), derived from the Aiguille Verte DEM, but a double-exponential distribution again gives a fairly close approximation.

Anisotropies in slope-forming processes can be expected to be reflected in anisotropies in the standard deviations of slope components in different directions. In North Wales, for example, the cirques are generally orientated towards the north-east (Evans, 1977). The area shown in Fig. 2(a), however, contains sufficient features with other aspects that the standard deviation varies little with direction, and similar values of $\sigma, 0.28$ and 0.29 respectively, are obtained for easterly and northerly slope components. The massive topography shown in Fig. 2(b), however, is dominated by large-scale features and is more anisotropic, with standard deviations varying between a maximum of 0.55 for northwest-south-east slope components (across the Chamonix valley axis) and a minimum of 0.5 for perpendicular directions.
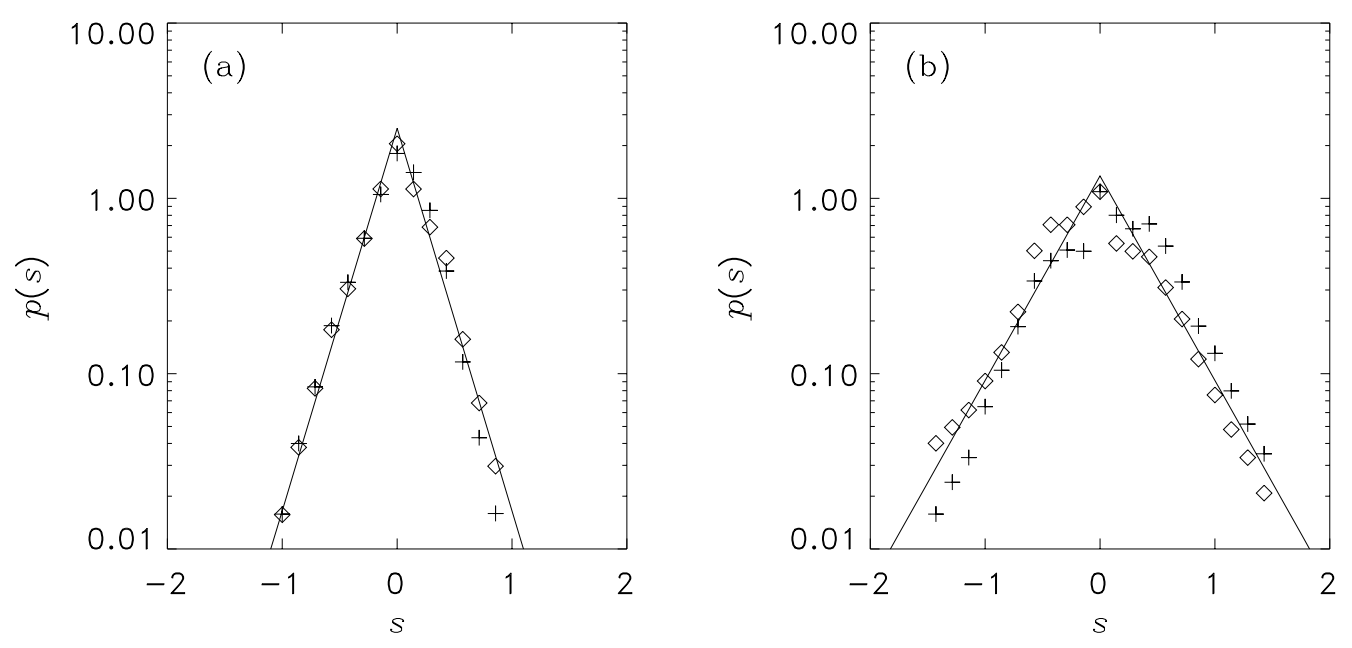

Fig. 3. Distributions of west-east (+) and south-north $(\diamond)$ slope components for the topographies shown in Figure 2, compared with Laplace distributions (lines). 
Slopes with $s<-\tan \theta$ are self-shaded for solar elevation $\theta$, so the fraction of the area that is self-shaded is found by integrating $p(s)$ over $s$ from $-\infty$ to $-\tan \theta$. For slope components following the distribution given by Eqn. (3), this fraction is

$$
f_{\text {self }}=\exp \left(-\frac{\sqrt{2}}{\sigma(\varphi)} \tan \theta\right),
$$

where $\sigma(\varphi)$ is specifically the standard deviation of slope components along transects in the direction of the solar azimuth. Because $\sigma$ is found to vary little with direction for the Snowdon DEM, a single value can be used for all azimuths. In Fig. 4(a), crosses show self-shaded fractions calculated by the terrain shading model and the dashed line shows predictions from Eqn. (4) for varying solar elevations and a fixed southerly azimuth; the agreement is good. Equation (4) also gives a good agreement with self-shaded fractions calculated for the Aiguille Verte DEM, as shown in Fig. 4(b), but the standard deviation of the slope component now has to be specified as a function of azimuth; for moderate anisotropy, this can be represented by an ellipse

$$
\sigma^{2}(\varphi)=\frac{\sigma_{+}^{2} \sigma_{-}^{2}}{\sigma_{+}^{2}-\left(\sigma_{+}^{2}-\sigma_{-}^{2}\right) \cos ^{2}\left(\varphi-\varphi_{+}\right)},
$$

where $\sigma_{+}$is the maximum standard deviation, $\varphi_{+}$is the azimuth at which it occurs and $\sigma_{-}$is the minimum standard deviation.

The angle from a point on the surface to its local horizon in some direction is the maximum angle from that point to any other point on the surface in that direction (Dozier and Frew, 1990). Points for which the sun lies below the horizon are in shadow. The fraction of the surface in shadow is thus equal to the cumulative probability that the horizon angle exceeds the solar elevation. Averaged over a large number of points, the distribution of maximum angles is given by the extreme value distribution

$$
f_{\text {shadow }}=1-\exp \left\{-\exp \left(-\frac{\theta-a}{b}\right)\right\},
$$

regardless of the shape of the distribution of angles between points on the surface (Gumbel, 1958). The parameters of Eqn. (6) are

$$
b=\frac{\sqrt{6}}{\pi} \sigma_{h}
$$

and

$$
a=\bar{h}-0.577 b,
$$

where $\bar{h}$ is the average and $\sigma_{h}$ is the standard deviation of the horizon angles determined by the terrain shading model. The predictions of Eqn. (6), shown by the solid lines in Figs. 4 (a) and (b), are again in good agreement with results from the terrain shading model (diamonds). Vertical bars show the range between minimum and maximum shadow fractions simulated for all solar azimuth angles; this range is small for simulations with the Snowdon DEM but is larger for low solar elevations with the Aiguille Verte DEM.

Shadow fractions are shown in Fig. 5 as functions of the time of day on 22 December (crosses) and 22 June (diamonds). For North Wales in summer, the entire area receives direct sunlight for most of the day according to the terrain shading model, although shadows will still be cast by boulders, vegetation and topographic features not resolved by the DEM. At the winter solstice, however, a
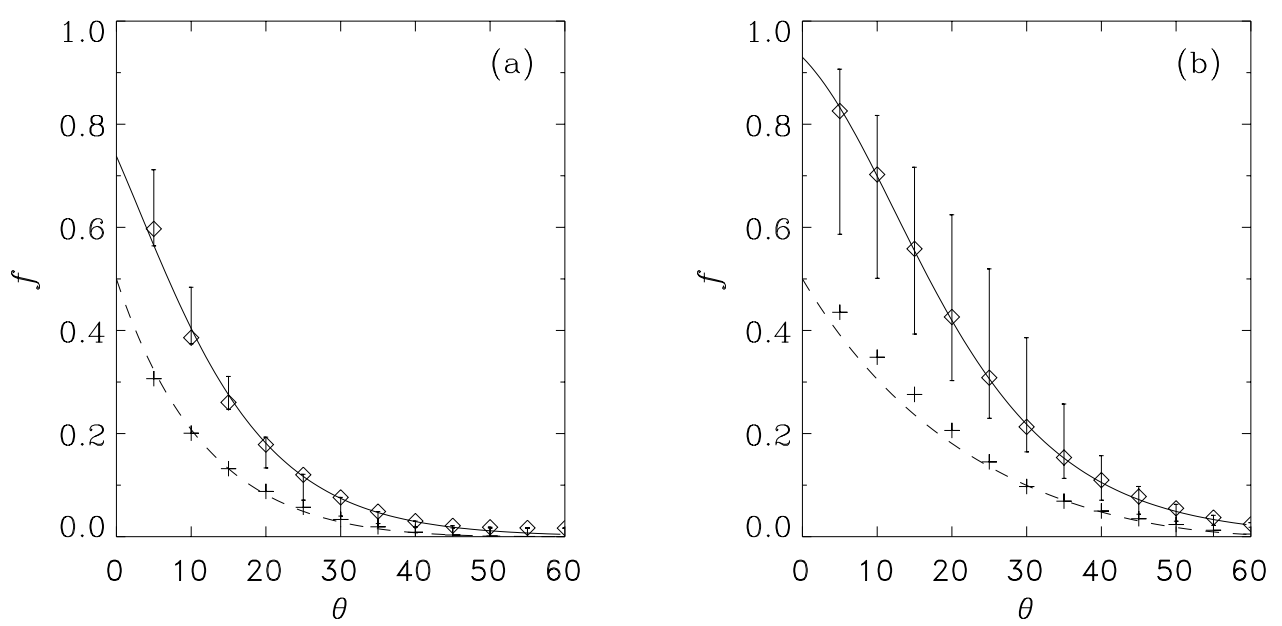

Fig. 4. Fractions of the areas in Figs. 2 (a) and (b) that are self-shaded (+) or horizon-shaded ( $\diamond)$ for illumination from the south with varying solar elevations. Dashed lines are from Eqn. (4) and solid lines are from Eqn. (6). Vertical bars show the range between minimum and maximum horizon-shaded fractions for all solar azimuth angles. 

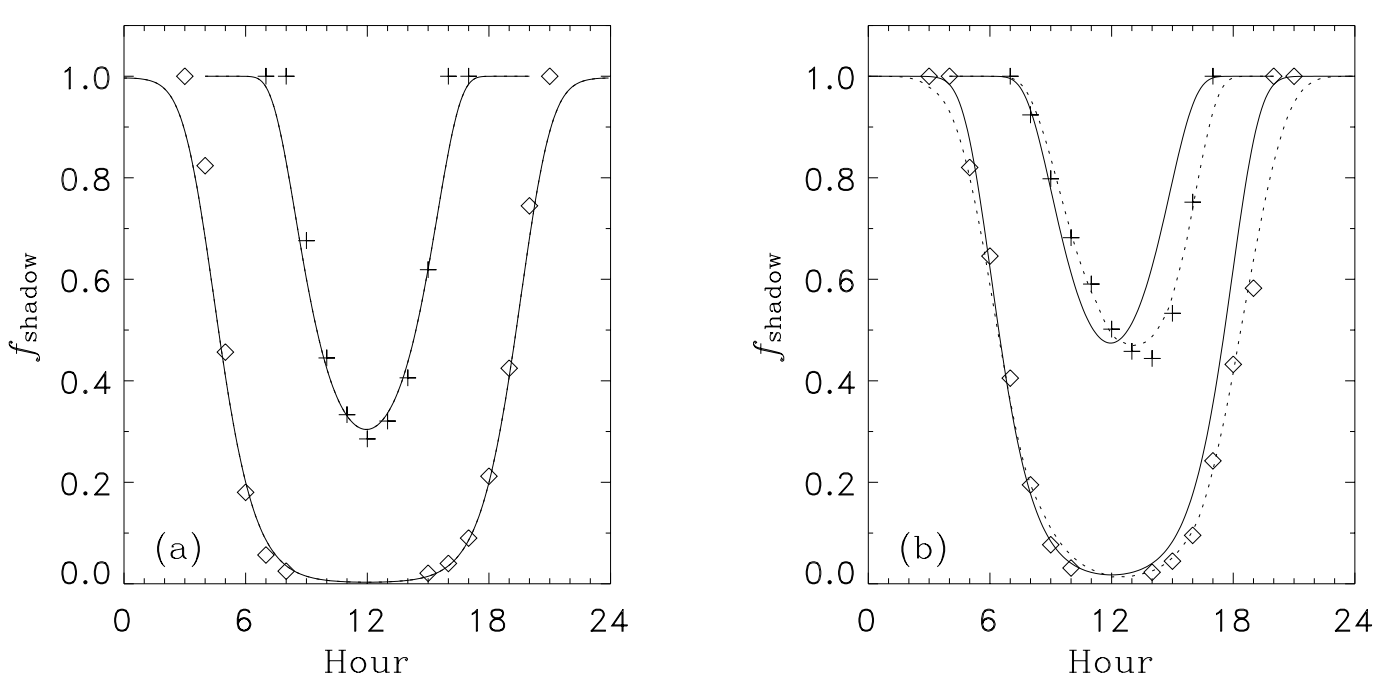

Fig. 5. Fractions of the areas in Figs. 2 (a) and (b) that are in shadow as functions of time on 22 December (+) and 22 June ( $\diamond)$. Solid lines are from Eqn. (6) with single values for the parameters, and the dotted lines in (b) were produced by fitting the parameters as functions of solar azimuth.

quarter of the area remains in shadow even at solar noon, with the sun rising only $14^{\circ}$ above the horizon. Equation (6) gives good fits to the simulation results for both dates and all azimuth angles with single values of the parameters $a$ and $b$; although Fig. 4 (a) shows that there is some variation in shadow fraction with azimuth for a fixed solar elevation, the range is small for the solar positions that actually occur. For the Aiguille Verte DEM, the terrain shading model gives shadow fractions that are noticeably asymmetric about solar noon at the winter solstice: $80 \%$ of the area is in shadow at 09:00, but only $53 \%$ is in shadow at 15:00, even though the sun has the same elevation $\left(10^{\circ}\right)$ at both times. This is because the broad valley running southwest to northeast in Fig. 2 (b) is shaded in the morning and sunlit in the afternoon. The symmetric curve given by Eqn. (6) with single values of the parameters gives a poor fit to the simulation results for low solar elevations, but better results can again be obtained by specifying $a$ and $b$ as functions of azimuth, as shown by the dotted lines on Fig. 5 (b).

\section{Conclusions}

For mountainous topography in North Wales and the French Alps, slope components were found to follow doubleexponential distributions. Knowledge of this distribution allowed the development of expressions giving the fractions of the surfaces that are self-shaded or in the shadow of remote topography for a given solar elevation and azimuth. These expressions were in good agreement with results from a terrain-shading model. In ongoing work, slope distributions for other regions are being investigated and used in the development of parameterisations of both directbeam and diffuse solar radiation for application in a statistical surface energy balance model.

Although the statistical expressions presented here were tested only for two areas, they are likely to have broader applicability. The double-exponential form of the distribution of slope components has been found to be a reasonable approximation for several other areas. An assumption of isotropy in values of the standard deviations of slope components over large enough areas, as found for North Wales but not for the more extreme topography of the French Alps, provides a useful but non-essential simplification. The extreme value distribution used to represent shadow fractions does not depend on the form of the underlying horizon angle distribution.

\section{Acknowledgements}

The Snowdon DEM was extracted from EDINA Digimap (Crown Copyright Ordnance Survey) by Krister Jansson. The terrain shading model and the Aiguille Verte DEM were supplied by Javier Corripio. Geomorphological information was provided by Mike Hambrey. Two anonymous reviewers made useful comments on this paper. The author is supported by NERC Advanced Research Fellowship NER/J/S/2001/ 00812 .

\section{References}

Barry, R.G., 1992. Mountain weather and climate. Routledge, London, UK. 402pp. 
Bowling, L.C., Pomeroy, J.W. and Lettenmaier, D.P., in press. Parameterisation of the sublimation of blowing snow in a macroscale hydrology model. J. Hydrometeorol.

Corripio, J.G., 2003. Vectorial algebra algorithms for calculating terrain parameters from DEMs and solar radiation modelling in mountainous terrain. Int. J. Geogr. Inf. Sci., 17, 1-23.

Dozier, J. and Frew, J., 1990. Rapid calculation of terrain parameters for radiation modelling from digital elevation data. IEEE Trans. Geosci. Remot. Sen., 28, 963-969.

Dubayah, R. and Rich, P., 1995. Topographic solar radiation models for GIS. Int. J. Geogr. Inf. Sci., 9, 405-419.

Dubayah, R., Dozier, J. and Davis, F.W., 1990. Topographic distribution of clear-sky radiation over the Konza Prairie, Kansas. Water Resour. Res., 26, 679-690.

Essery, R.L.H., 2001. Spatial statistics of windflow and blowing snow fluxes over complex topography. Bound.-Lay. Meteorol., 100, 131-147.

Evans, I.S., 1977. World-wide variations in the direction and concentration of cirque and glacier aspects. Geogr. Ann., 59A, $151-175$.
Garnier, B.J. and Ohmura, A.,1968. A method of calculating the direct shortwave radiation income of slopes. J. Appl. Meteorol., 7, 796-800.

Gumbel, E.J., 1958. Statistics of extremes. Columbia University Press, New York, USA. 375pp

Kumar, L., Skidmore, A.K. and Nowles, E.K. 1997. Modelling topographic variation in solar radiation in a GIS environment. Int. J. Geogr. Inf. Sci., 11, 475-497.

Mckenney, D.W., Mackey, B.G. and Zavitz, B.L.,1999. Calibration and sensitivity analysis of a spatially-distributed solar radiation model. Int. J. Geogr. Inf. Sci., 13, 49-65.

Oliphant, A.J., Spronken-Smith, R.A., Sturman, A.P. and Owens, I.F., 2003. Spatial variability of surface radiation fluxes in mountainous terrain. J. Appl. Meteorol., 42, 113-128.

Ranzi, R. and Rosso, R., 1995. Distributed estimates of incoming direct solar radiation over a drainage basin. J. Hydrol., 166, $461-478$.

Skidmore, A.K., 1989. A comparison of techniques for calculating gradient and aspect from a gridded digital elevation model. Int J. Geogr. Inf. Sci., 3, 323-334. 